\title{
Adsorption of gas-like molecules to self-aligned square-well fluid channels under confinement of chemically patterned substrates
}

\author{
Satya Pal Singh $\cdot$ Jayant K. Singh • \\ Ashutosh Sharma
}

Received: 21 October 2011/Accepted: 7 April 2012/Published online: 24 May 2012

(C) The Author(s) 2012. This article is published with open access at Springerlink.com

\begin{abstract}
To extend the work of binary fluid mixtures and their associated bridge-like structures, the adsorption of gas-like molecules (interacting via hard-sphere potentials) on self-assembled fluid channels was examined. We examined the morphological evolution of an initial random binary mixture under confinement of chemically patterned substrates with strong, long-range preferential attraction to the pure square-well component. Gas-like molecules were presumed to have a weak attraction to the square-well fluid. The morphology and corresponding density profiles revealed the underlying chemical and physical adsorption of gas-like molecules to off-strip voids and to the interface of the self-assembled fluid channels. The entropic effects drive the non-interaction hard-sphere molecules to assemble or reorganize in the voids left between the selfassembled square-well fluids. Such studies can help in the study of formation of nano-liquid structures and enhanced adsorption of gas-like molecules for storage purposes.
\end{abstract}

Keywords Square-well fluid · Hard spheres · Entropic effect - Chemical and physical adsorption

The work was presented by Dr. S. P. Singh at the second International Conference on Advanced Nanomaterials and Nanotechnology, ICANN-(8-10 Dec. 2011) in oral session H-1638.

\section{Present Address:}

S. P. Singh $(\varangle)$

Department of Applied Sciences, Madan Mohan Malviya Engineering College, Gorakhpur 273010, Uttar Pradesh, India e-mail: singh.satyapal@hotmail.com

\section{S. P. Singh · J. K. Singh $(\bowtie) \cdot$ A. Sharma}

Department of Chemical Engineering, Indian Institute of Technology Kanpur, Kanpur 208016, Uttar Pradesh, India e-mail: jayantks@iitk.ac.in

\section{Introduction}

Theoretical and simulation studies have demonstrated the formation of a bridge phase in both one- (Schoen 2008; Trokhymchuk and Alejandre 1999) and two-component (Yuan et al. 2004; Herrera et al. 2005) fluids near a twophase co-existence confined by patterned and unpatterned surfaces. In the one-component case, the bridge phase consists of alternating regions of liquid and vapor. In the two-component case, the bridges are alternated with the two components. Recent attention has focused on the influence of surface-induced transitions on the net force acting between the plates (Overduin and Patey 2002). Bridge-phase formation leads to strong attractive plateplate forces that are equal in magnitude to those observed for homogeneous surfaces. Simulation techniques are important in such research when it becomes difficult to perform experiments at very small scales.

Phase separation and related wetting phenomena (Puri 2007; Yan et al. 2008) for binary fluid mixtures in contact with an adsorbing surface have attracted considerable attention. Such phenomena occur in a binary liquid mixture below the consolute point where one phase is adsorbed on the wall that has preferential attraction with the component. The growth dynamics of fluid layers adsorbed on channels have been investigated experimentally (Lipowsky 2001; Fukuzawa et al. 2008; Kargupta and Sharma 2002), and the wetting of rings on nano-structured patterns have been observed using Monte Carlo (MC) simulation (Porcheron et al. 2006). When liquids are adsorbed in nanopores or capillaries, their properties are markedly changed from those in bulk (Christenson 2001; Gelb et al. 1999; Rother et al. 2004). Confinement shifts the first-order wetting transitions. Sometimes, surface-induced phase transitions such as layering and prewetting compete with shifted bulk 
transitions, leading to rich-phase diagrams. The morphologies of the intervening wetting structure reflect the underlying chemical and physical patterning of the substrates (Christenson 2001).

Consider the example of a liquid phase $\beta$ close to twophase co-existence with another fluid phase $\alpha$ and confined within a slab between two solid substrates. If both surfaces are homogeneous, the surface will either be wetted or dewetted by the $\beta$ phase, depending on the preferential attraction of the surfaces. If the surfaces contain two types of domains $\delta$ and $\gamma$ that repel and attract the $\beta$ phase, respectively, then the $\beta$ phase will seek to maximize its contact with the $\gamma$ domains on both surfaces while seeking to avoid the $\delta$ regions (Lipowsky 2001). The simplest pattern of surface domains consists of a single pair of opposing lyophilic strips (Fig. 1a-c) separated by a distance $H$. Two $\gamma$ strips of width $L \gamma$ lie parallel to each other, but may be displaced by $\Delta L$ (Fig. 1c). When a certain amount of $\beta$ phase is placed within the structured slab with a sufficiently small $H$, the liquid will form a bridge connecting the two $\gamma$ strips (Fig. 1a, b). For larger values of $H$, the bridge will break and form two separate channels (Fig. 1c).

Trazona et al. (Rocken and Tarazona 1996) previously presented phase diagrams for capillary condensation in chemically structured pores. Working with a complex periodic wall potential in one of the lateral dimensions, they observed that liquid drop condensation at the most attractive adsorption site led to the repeated formation of liquid bridges between opposite walls separated by a gas (Fig. 2). Employing non-local density functional theory to investigate the stepwise mechanism and the liquid bridgephase stability, they found that the maximum lengths of the liquid bridges were predominantly limited by the associated liquid-liquid interfacial tension. For striped patterns, bridges are only observed when the pore width $H$ is less than or roughly equal to the strip width $\xi$ (Schoen 2008).
GCMC calculations indicate that Lennard-Jonnes interactions with a small amount of surfactant exhibit enhanced bridge formation. This is because the surfactant reduces the unfavorable liquid-liquid interfacial tension, thereby reducing the interfacial energy between the two components when a bridge is formed (Elliott and Hu 1999).

In addition to extending the range of liquid bridges, use of a combination of chemically patterned surfaces and surfactants can generate other structures if the liquid mixture is near demixing co-existence. When the surfaces are too distant for bridges to exist, extended bubbles of a particular phase form adjacent to the attractive surfaces. These structures can be essentially viewed as surface-induced "micelles", and their shape depends on the geometry of the attractive surface patches. Formation of repeated liquid bridges has also been reported.

The adsorption of gas-like molecules (presumably interacting via hard-sphere potentials) on self-assembled fluid channels has only rarely been explored experimentally or computationally, through the morphological evolution of an initial random binary mixture under confinement of chemically patterned substrates. Nevertheless, this scenario is important from the perspective of gas storage or enhancing gas-like molecular adsorption in nano pores or cavities (e.g., carbon nanotubes, zeolites, etc.). Indeed, hydrogen storage for fuel cells has been a challenging problem in the recent past (Darkrim and Levesque 1998), with researchers attempting doping techniques to optimize hydrogen storage in nano-structured cavities (Roussel et al. 2009; Cabria et al. 2005; Bhatia and Myers 2006). A further advancement in this direction can help in the study of solid-liquid transition and the formation of multi-component matrix near substrates using other simulation techniques as SGMC (semi grandcanonical Monte Carlo) which can help in exploring the problems of heat energy storage and selective solar absorption by tandem etc. (Lee Kil Dong 2010; Katumba et al. 2008a, b).
Fig. 1 a In bridge, b out bridge, and $\mathbf{c}$ channel liquid and gas phases

Fig. 2 In bridge structures with
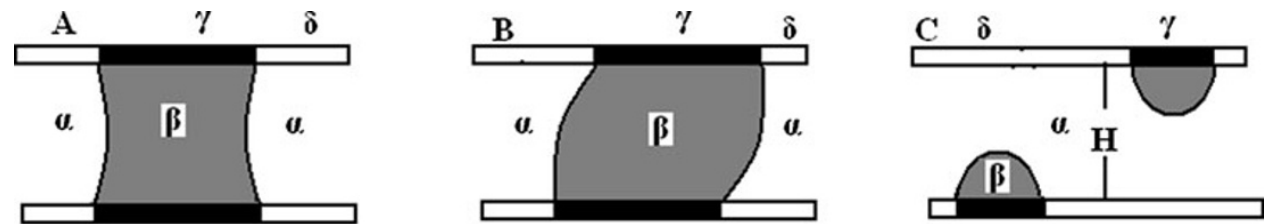

$\gamma$

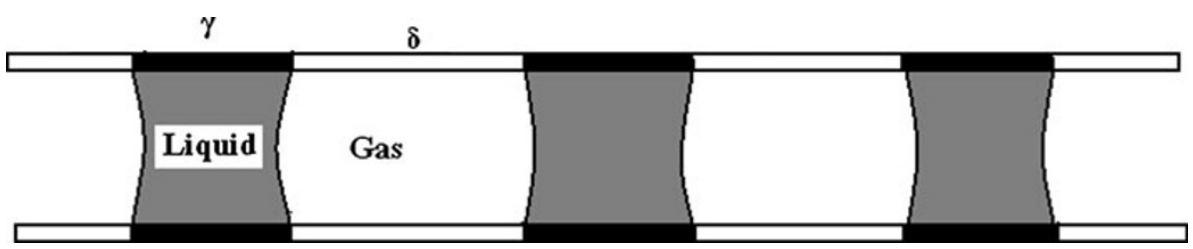




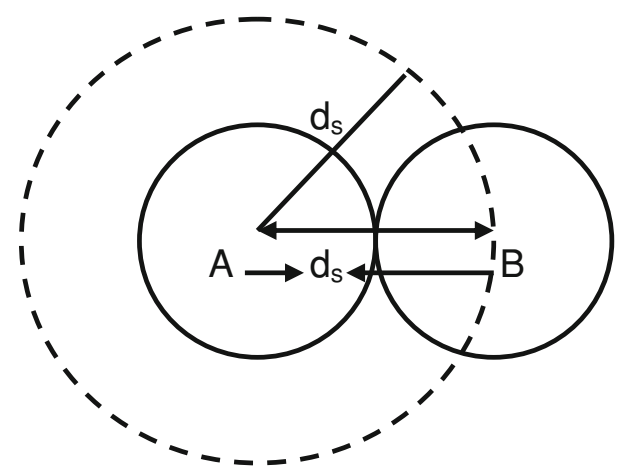

Fig. 3 The excluded volume: two hard spheres each of radius $r$

\section{Entropic effect and the modeling}

Entropic interactions and the entropic force in solutions

\section{Excluded volume of a sphere}

The excluded volume makes the real particle-particle or monomer interactions non-ideal. Let's first consider the case of two hard spheres A and B of radius $r$ each Fig. 3. The center to center distance between spheres A and B cannot be less than $d_{\mathrm{s}}$. Sphere B is excluded by sphere A. The space not available to the center of $\mathrm{B}$ is a sphere of radius $d_{\mathrm{s}}$ (i.e., $d_{\mathrm{s}}=2 r$ ) as indicated by dashed lines. Thus, the excluded volume is

$v_{\mathrm{e}}=\frac{4}{3} \pi d_{\mathrm{s}}^{3}=\frac{4}{3} \pi(2 r)^{3}=8 v$

$v_{\mathrm{e}}=8 \times$ volume of one sphere

Now, let us consider a process in which the volume excluded by each of the two spheres increases from $0 \rightarrow v_{\mathrm{e}}$. The space available to the other sphere decreases from total volume $V$ of the system to $V-v_{\mathrm{e}}$. Thus, the configurational entropy of the sphere changes by

$$
\begin{aligned}
& \Delta S=k_{\mathrm{B}} \ln \frac{V-v_{\mathrm{e}}}{V}=k_{\mathrm{B}} \ln \left(1-\frac{v_{\mathrm{e}}}{V}\right) \\
& \Delta S=-k_{\mathrm{B}} \frac{v_{\mathrm{e}}}{V} \text { for } v_{\mathrm{e}} \ll V
\end{aligned}
$$

From first-order expansion, the change in the Helmholtz free energy is then

$\frac{\Delta A}{k_{\mathrm{B}} T}=-\frac{\Delta S}{k_{\mathrm{B}}}=\frac{v_{\mathrm{e}}}{k_{\mathrm{B}}}$

When the system has $N$ identical spheres in the volume of $V$, there are $\left[{ }^{N} \mathrm{C}_{2}=N(N-1) / 2\right]=N^{2} / 2 f$ pairs of excluded-volume interaction. Now, the change per sphere in total free energy due to excluded volume is

$\frac{\Delta A}{N k_{\mathrm{B}} T}=\frac{N v_{\mathrm{e}}}{2 V} \propto \frac{N}{V} \equiv$ density

At low concentrations, the excluded volume and the contribution in free energy change by excluded volume is low. As the concentration increases and $N v_{\mathrm{e}} \rightarrow V$, the excluded-volume effect becomes very strong. Important to note here that in solutions, the excluded volume does not disappear even in low concentration limit. Particle sizes make the situation different from that in the suspension of spheres. Let us consider a solution consisting of $N$ spheres of diameter a. Here we have not considered particle-particle interactions for the sake of clarity in understanding the entropic effects only. These $N$ spheres or monomers in case of polymers can be considered to be contained in a cube of volume $R^{3}$.

The Helmholtz free energy $A$ change per chain due to the excluded volume is

$\frac{\Delta A}{k_{\mathrm{B}} T}=-\frac{\Delta S}{k_{\mathrm{B}}}=\frac{N^{2} v_{\mathrm{e}}}{2 R^{3}}$

This is originated from entropic consideration. This excluded volume can be regarded as an interaction and we can write

$v_{\mathrm{e}}=\frac{4}{3} \pi d_{\mathrm{s}}^{3}=\frac{4}{3} \pi(2 r)^{3}=\frac{32}{3} \pi r^{3}$

$\frac{\Delta A}{k_{\mathrm{B}} T}=\frac{N^{2}}{2} \frac{32}{3} \frac{\pi r^{3}}{R^{3}}$

$\frac{\Delta A}{k_{\mathrm{B}} T} \propto \frac{N^{2}}{R^{3}}$ because $\frac{N^{2}}{R^{3}}=\left[\frac{N}{R^{3}}\right]^{2} R^{3}$

$N^{2} / R^{3}$ is equal to the volume $R^{3}$ multiply by the square of the particle or monomer density (i.e., $N / R^{3}$ ). It establishes the fact that even a particle-particle noninteracting system will experience the force driven by the entropic contribution to the system (Singh 2008). It can lead even hard sphere system with same or different size particles to reorganize themselves in different confirmations for minimum free energy of the system.

Brief theory

There are two possible final configurations for a binary mixture of fluids confined between two planar walls. These configurations are complete wetting $(\mathrm{CW})$ and partial wetting (PW), and depend on the strength of attraction between the A molecules and the two walls as well as on the separation of the two walls, $L_{\mathrm{z}}=H$. The equilibrium configuration arises as a consequence of the minimization of the total interfacial free energy.

When the attraction between the two walls and the water-like particles are strong, the water particle completely wets the two walls. For the $\mathrm{CW}$ configuration, the interfacial free energy in 3D can be written following (Yuan et al. 2004):

$F_{\mathrm{CW}}=\gamma_{\text {wall-A }} S_{\text {wall-A }}+\gamma_{\mathrm{AB}} S_{\mathrm{AB}}$

Here, $S_{\text {wall-A }}$ is the total interface area of wall-A interfaces, $S_{\mathrm{AB}}$ is the area of the A-B interface, and $\gamma_{\text {wall-A }}$ and $\gamma_{\mathrm{AB}}$ are 
the interfacial free energies per unit area between the wall and $\mathrm{A}$ and between $\mathrm{A}$ and $\mathrm{B}$, respectively. The structures evolve to minimize $F_{\mathrm{CW}}$. When the concentration of particle $\mathrm{A}$ is less than a certain critical value (i.e., 0.682 for the $2 \mathrm{D}$ case (Yuan et al. 2004)), the A particles are divided into two parts. Each part layers on the walls and gives rise to $\mathrm{CW}$. When the concentration of pure component $\mathrm{A}$ is more than the critical value, the $\mathrm{B}$ particles become concentrated in globoid form. In this case, the A particles occupy the residual space and completely wet the two walls.

The above scenario has been studied with two squarewell fluid mixtures of $\mathrm{A}$ and $\mathrm{B}$ components. When the attraction between the two walls and water is weak or intermediate, the A particles form a bridge or concave lenslike structure. In the PW case, the total interfacial energy can be given by

$$
\begin{aligned}
F_{\mathrm{PW}}= & \gamma_{\text {wall-A }} S_{\text {wall-A }}+\gamma_{\text {wall-B }}\left(2 L_{x} \times L_{y}-S_{\text {wall-A }}\right) \\
& +\gamma_{\mathrm{AB}} S_{\mathrm{AB}},
\end{aligned}
$$

where $\gamma_{\text {wall-A }}, \gamma_{\text {wall-B }}$ and $\gamma_{\mathrm{AB}}$ are the interfacial energies between wall-A, wall-B, and A-B species, respectively. We observed the different phases in $3 \mathrm{D}$, and assumed that when one of the lateral dimensions (i.e., $y$ ) is kept large, then the volume can be thought of as being composed of different $2 \mathrm{D}$ slabs. A large lateral direction generates patterns that are homogenous in the $y$ direction. The dimensional effect does not alter the qualitative phenomenological behavior. A formal evaluation of the pressure tensor components and corresponding surface tensions for square-well fluids can be found in Orea et al. (2003) and Singh and Kofke (2005).

\section{Model and simulation geometry}

Canonical Monet Carlo (NVT-MC) simulations were performed in cells of dimension $L_{x} \times L_{y} \times\left(L_{z}=h\right)$. Periodic boundary conditions were used in the $X$ and $Y$ directions, and two planar surfaces of infinite thickness were placed at $z=0$ and $z=h$.

The fluid mixture contains a liquid-like component $\mathrm{A}$ and a gas-like component B. Square-well (Orea et al. 2004; Singh et al. 2003; Green et al. 1994) fluids have been extensively studied by computer simulations and statistical methods. The square-well potential contains the essential features of repulsion and attraction (Fig. 4). We considered the fluidfluid interaction as a square-well interaction between particles of type $i$ and $j$ (A and $\mathrm{B}$ ), as defined below.

$u_{i j}(r)=\left\{\begin{array}{ll}\infty & \text { if } r<\sigma_{i j} \\ -\epsilon_{i j} & \text { if } \sigma_{i j} \leq r<\lambda_{i j} \sigma_{i j} \\ 0 & \text { otherwise }\end{array}\right\}$

Here $\sigma_{\mathrm{ij}}$ and $\varepsilon_{\mathrm{ij}}$ are the length and energy parameter, respectively. $\lambda_{\mathrm{ij}}$ defines the interaction range. The strength

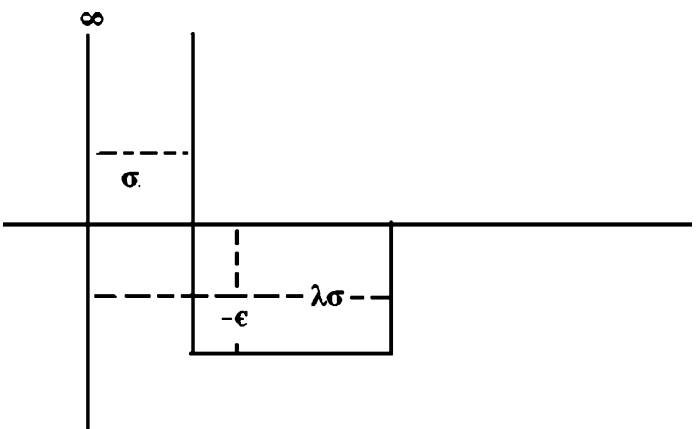

Fig. 4 Sketch of a square-well potential

of the interactions is determined by $\varepsilon_{i j}$. The potential form of the above equation is shown in Fig. 4. We selected $\varepsilon_{\mathrm{AA}}=1.0, \varepsilon_{\mathrm{BB}}=0.0, \varepsilon_{\mathrm{AB}}=0.5$, and $\lambda_{i j}=1.5$ for all cases, with initial average number densities of $\rho=0.40$, 0.50 , and 0.60 . We assume that $L_{x}=L_{y}, L_{z}=4.0$ for $\rho=0.40$, and $L_{z}=8.0$ for $\rho=0.50,0.60$. We also assume that the concentration ratio of $\mathrm{A}: \mathrm{B}$ is $50: 50$. The number of A and B molecules is 800 each with $\rho=0.40$, and 400 each for $\rho=0.50,0.60 . \sigma_{i j}=1.0$ is taken as the hard core radius for all $i$ and $j$. Thus, we studied the additive binary mixture $\sigma_{\mathrm{AB}}=\left(\sigma_{\mathrm{A}}+\sigma_{\mathrm{B}}\right) / 2$.

We did not impose a long-range energy correction to the particle-particle square-well interaction (Grosfils and Lutsko 2009), under the assumption that it would not change the qualitative behavior. The model was simplified following the results of recent simulation studies. We sought to determine the 3D channel-like morphological evolution of self-assembled structures of an initial random binary mixture of square-well-hard-sphere particles confined between two parallel plates. The particles align along chemical patterns on the substrates near demixing co-existence. Canonical ensemble MC simulation was used to observe the separation and adsorption of gas-like molecules (which interact by pure hard-sphere potentials) on the self-assembled channels, inside pores, or within created voids. Such a growth process of wetting layers has been reported for binary liquid mixtures (Steiner and Klein 1996). Particles of species A, with centers at a distance $Z$ from the wall, interact with each surface through the potential

$u_{i}^{\text {wall }}(z)=\left\{\begin{array}{ll}\infty, & \text { if } z<\frac{1}{2} \sigma_{i} \\ -\varepsilon_{i}^{\text {wall }}, & \text { if } \frac{1}{2} \sigma_{i} \leq z<\lambda_{i} \sigma_{i} \\ 0, & \text { otherwise }\end{array}\right\}$

In the present study, $\varepsilon_{\mathrm{A}}^{\text {wall }}=3.0$ with $\lambda_{\text {wall-A }}=2.0$ was assumed for all cases. B molecules have hard-sphere interactions with surfaces.

The starting configuration consisted of cubes of A and B phases in two halves of the volume. The system was melted at a high temperature of $T=12.0$ for $10^{5}$ cycles. Next, the 
system was equilibrated at $T=0.80$ for $20 \times 10^{5}$ cycles, with the maximum displacement $\chi_{i}^{\max }$ changing every 10 cycles to achieve a $50 \%$ acceptance ratio (Frenkel and Smit 1996; Allen and Tildesley 2001) during the equilibration period for $N=800$ and $\rho=0.50,0.60$. The simulation was employed for only particle displacements. After equilibration, the maximum displacement $\chi_{i}^{\max }$ was fixed and the system was simulated for $40 \times 10^{5} \mathrm{MC}$ cycles, with $N=800$ and $\rho=0.50,0.60$. For $N=1,600$ and $\rho=0.40$, the equilibration and production cycles were taken as $10 \times 10^{5}$ cycles and $20 \times 10^{5}$ cycles, respectively, to reduce computational cost. Density and pressure values were sampled every 1,000 cycles after equilibration. Following the Metropolis algorithm, we accepted a particle displacement of $r^{\prime}=r+\Delta r$, where $\Delta r$ was randomly chosen such that $-\chi_{i}^{\max } \leq \Delta \chi_{i} \leq \chi_{i}^{\max }$ where $i=1,2$, and 3 for the $x, y$, and $z$ coordinates, respectively.

\section{Results and discussion}

Density profiles in the $x$ direction perpendicular to the interfaces are shown in Fig. 5a-c for chemically patterned strips with strip widths of $\delta=6.0,8.0$, and 10.0, respectively. In this example, we used a temperature $T=0.80$ for a simulation box with $L_{Z}=H=4.0$ and a total average number density $\rho=0.40$. Rich regions of type A molecules were observed along the strip surfaces. This type of onecomponent enrichment on chemically patterned channels has been reported previously (Lipowsky 2001; Fukuzawa et al. 2008; Kargupta and Sharma 2002), but only with single fluids. In the present study, the gas-like molecules were dominantly separated to off-strip regions. Thus, fluid channels of the A component self-assembled along the attractive strips, as distinctly indicated by the morphological evolution micrographs (Fig. 6).

Gas-like molecules B had weak attractive square-well interactions with intermediate-range liquid-like molecules $\mathrm{A}$, defined by interaction parameters $\lambda_{\mathrm{AB}}=1.5$ and $\varepsilon_{\mathrm{AB}}=0.5$. Once these fluid channel morphologies evolved with component A, a (rough) interface of A-B appeared on which $\mathrm{B}$ molecules became absorbed because of its weak attractive potential with $\mathrm{A}$. In the density distribution, two small by distinct peaks of gas-like molecules near A enriched the channel regions. These peaks formed as a result of the weak attractive potentials of A and B. The area between the self-assembled channels showed flat regions in the density curves of $\mathrm{B}$. These regions corresponded to the non-bonding accumulation of gas-like molecules in the pores or voids formed from the morphological evolution of channel-like structures.

A surprising phenomenon was found in the $z$-density profile of Fig. 5d. Although the strips had strong,
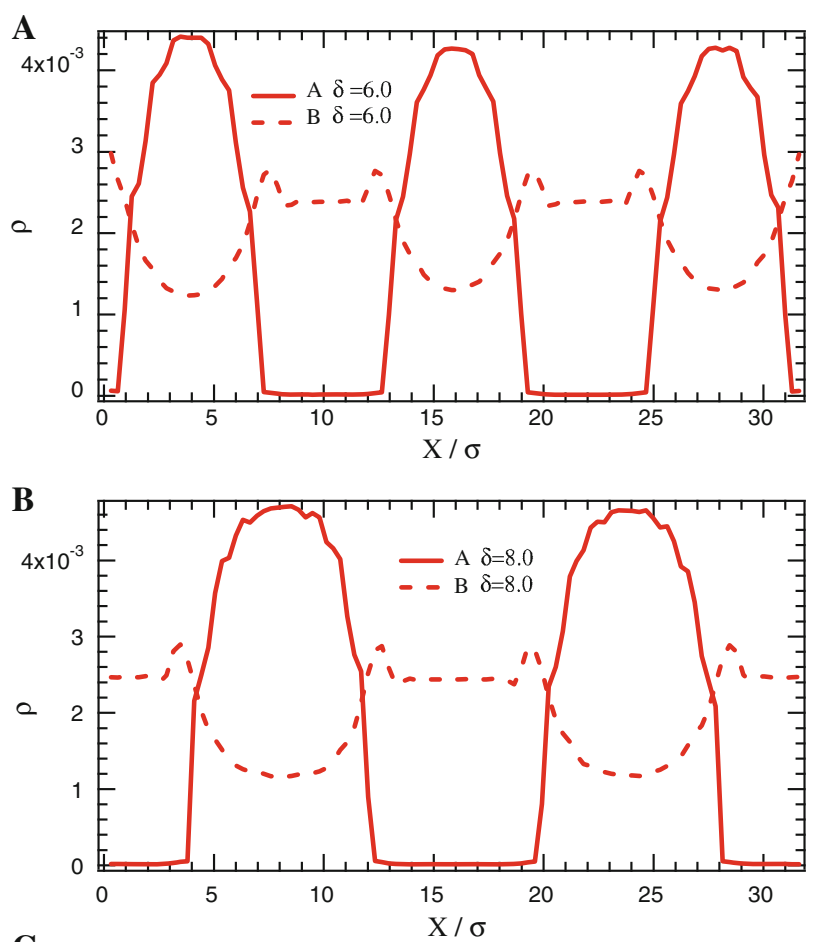

C

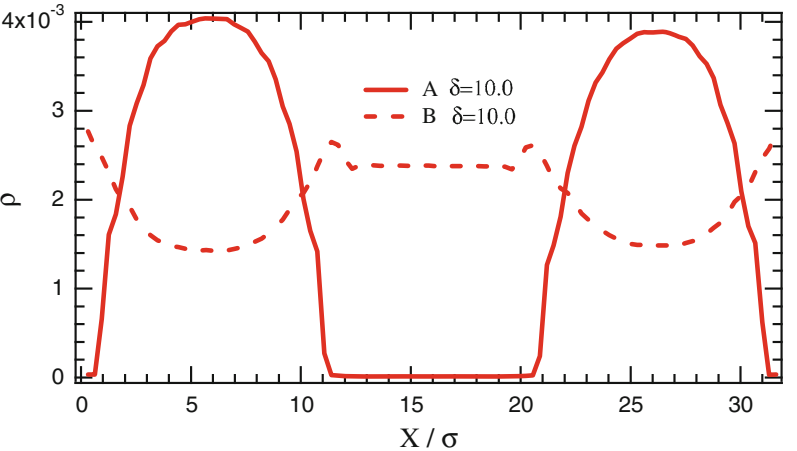

D

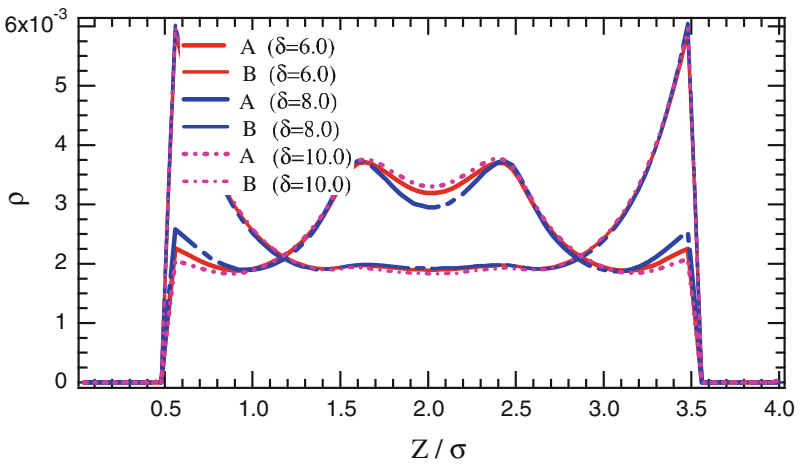

Fig. 5 Density profiles of A (square well) and B (hard sphere) types in the $x$ direction with a strip width of $\delta=6.0(\mathbf{a}), \delta=8.0(\mathbf{b})$, or $\delta=10.0$ (c), and in the $z$ direction at a reduced temperature of $T=0.80$ (d). Strip widths are shown in parentheses. Substrates have a long-range attractive square-well potential with A. Solid and dashed lines correspond to $\mathrm{A}$ and $\mathrm{B}$ types, respectively. An initial total average number density of $\rho=0.40$, slit width of $H=4.0$, and composition ratio of $\mathrm{A}: \mathrm{B}=50: 50$ are assumed. The different interaction parameters were: $\lambda_{\mathrm{AA}}=1.5$ and $\varepsilon_{\mathrm{AA}}=1.0 ; \lambda_{\mathrm{AB}}=1.5$ and $\varepsilon_{\mathrm{AB}}=0.5 ;$ and $\lambda_{\text {wall-A }}=2.0$ and $\varepsilon_{\text {wall-A }}=3.0$ 

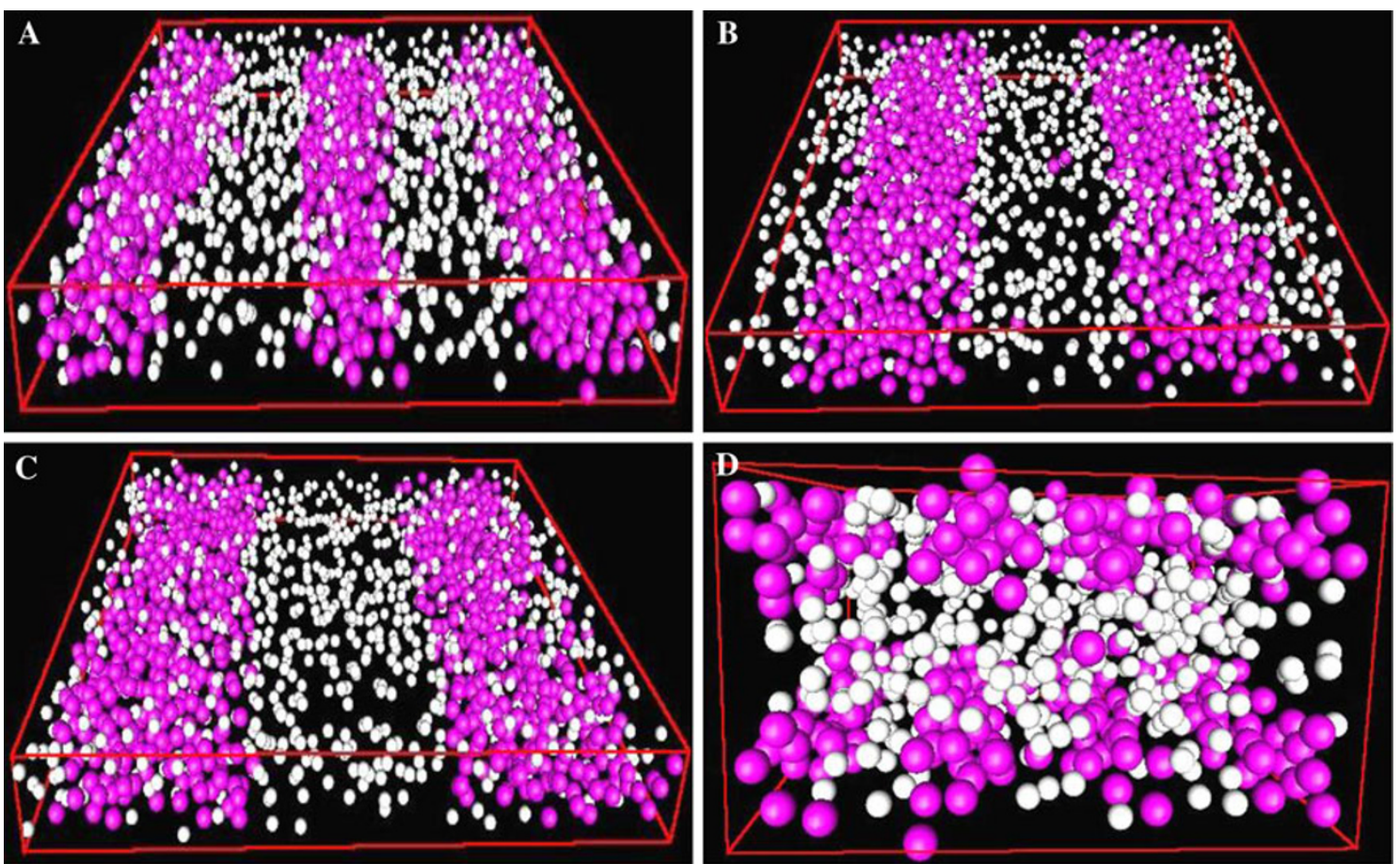

Fig. 6 Micrograph with strip widths of $\delta=6.0,8.0$, or 10.0 from left to right for $\mathrm{H}=4.0, \mathrm{~T}=0.80$, and $\rho=0.40$. Bottom right corner micrograph shows configuration for $\delta=2.0$ with $\mathrm{H}=8.0, \mathrm{~T}=0.80$,
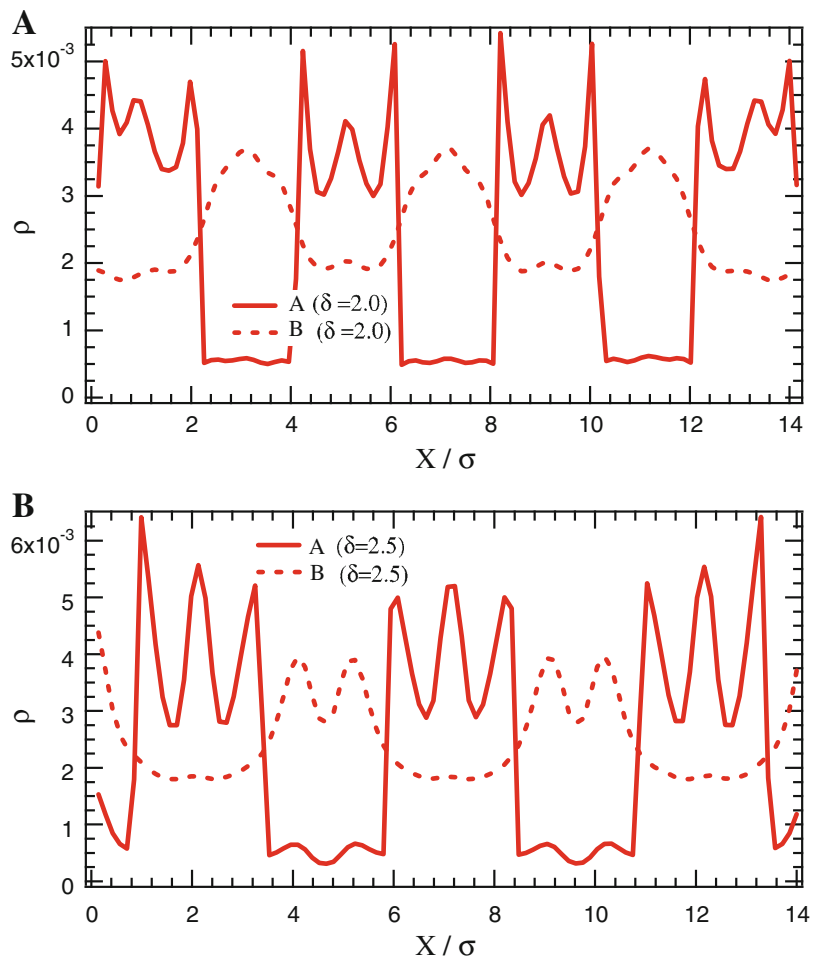

Fig. 7 Density profiles of A (square well) and B (hard sphere) types in the $x$ direction with a strip width of $\delta=2.0$ (a) or $\delta=2.5$ (b). Substrates have a long-range attractive square-well potential with A. Solid and dashed lines correspond to A and B types, respectively. $\rho=0.50$ and $\mathrm{A}: \mathrm{B}=50: 50$ were used. The different interaction parameters were the same as in Fig. 5

and $\rho=0.50 . \mathrm{A}: \mathrm{B}=50: 50$ in all cases. The different interaction parameters are the same as in Fig. 5

long-range attractive square-well interactions with A compared to the pre-component interaction of A-A, the average density of A peaked near $H=2.0$ rather than near the substrates. Twin peaks were observed in the mid-region of the $z$-density profile for component A where the two surface fields of the substrates overlap. This enrichment in the middle of the simulation box further attracted more molecules of type A, creating a curve (Fig. 5d). Since the strip widths of $\delta=6.0,8.0$, and 10.0 were greater than $L_{Z}=H=4.0$, the scenarios in Fig. 5 correspond to the case of two-drop formation on substrates. These drops can be merged to form a bridge-like structure when the separation between the two plates $H$ is small with a relatively denser region in the middle.

Simulation results with narrower strips are shown in Figs. 7, 8, and 9. These simulations were performed with a larger slit width $\left(L_{Z}=H=8.0\right)$. The total average number densities were $\rho=0.50$ and 0.60 with attractive strip widths of $\delta=2.0$ and 2.5 at a temperature of $T=0.80$. The qualitative behavior of the morphological evolution of the square-well hard-sphere mixture was similar to that described above, except that the narrower channels generated $W$-shaped peaks in the $X$ density profile of component A. These peaks arose from the molecular rearrangement of A-type molecules on attractive strips of widths of $2.0 \sigma$ and $2.5 \sigma$ (molecular diameter $\sigma=1.0$ ). When the strip width was set to $\delta=2.0$, the density curves corresponding to the 

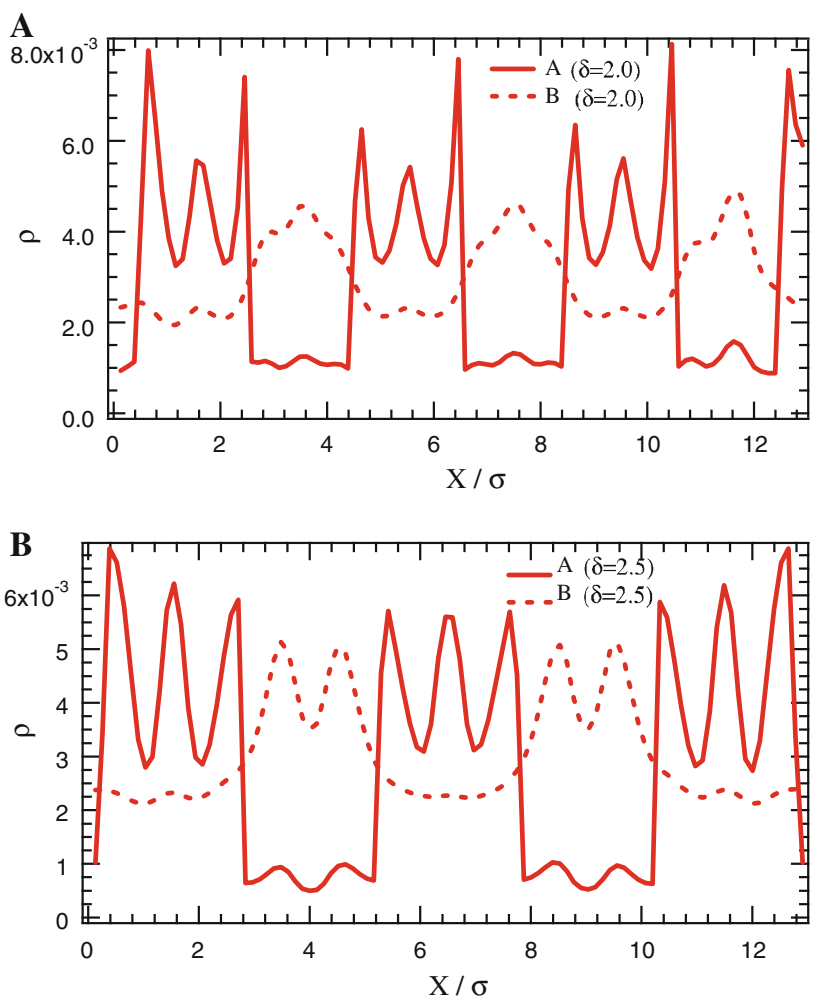

Fig. 8 Density profiles of A (square well) and B (hard sphere) types in the $x$ direction with a strip width of $\delta=2.0$ (a) or $\delta=2.5$ (b). Substrates have a long-range attractive square-well potential with A. Solid line and dashed lines correspond to A and B types, respectively. $\rho=0.60$ and $\mathrm{A}: \mathrm{B}=50: 50$ were used. The different interaction parameters were the same as in Fig. 5

gas-like molecules B showed single peaks in the off-strip regions or voids created by channel formation by the pure square-well component A. In contrast, a strip width of $\delta=2.5$ generated two distinct peaks in the void regions (Figs. 7, 8). This behavior was more pronounced in cases with a higher average total number density $(\rho=0.60)$. The $z$-density profiles for Fig. 9 indicate that the attractive strip regions on the two substrates caused a thick layering of the A component near the substrates along the strips, generating channel-like structures. The unlayered regions were occupied by gas-like molecules $\mathrm{B}$.

\section{Conclusion}

The computational model presented here can successfully predict and explain the adsorption of gas-like molecules on self-assembled fluid channels and their reorganization in different confirmations of rod like and compact spheres between the voids when the initial system evolves from an initial random mixture of square wells and hard spheres. The model also provides morphological details and possible engineering, even though no optimized study of
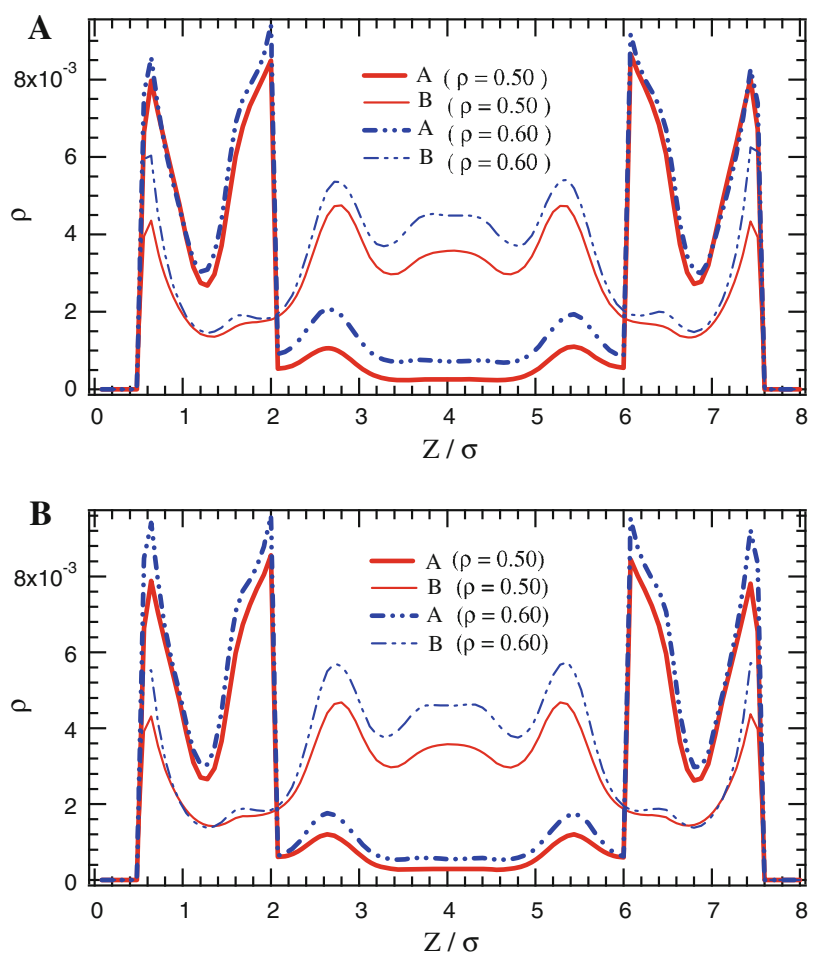

Fig. 9 Density profiles of A (square-well) and B (hard sphere) types in the $z$ direction with a strip width of $\delta=2.0$ (a) or $\delta=2.5$ (b). Substrates have a long-range attractive square-well potential with A. Solid and dashed lines correspond to A and B types, respectively. The initial total average number densities are shown in parentheses. $\mathrm{A}: \mathrm{B}=50: 50$. The different interaction parameters are the same as in Fig. 5

adsorption was performed. When the simulations were performed with a strong A-B interaction (e.g., $\varepsilon_{\mathrm{AB}}=1.0$ ), the mixing of the two components reduced the difference between their peak values in the density distribution. However, these results are not shown in the manuscript because the qualitative behavior was unchanged by the stronger interaction. Figure 10 (Singh et al. 2011) demonstrates the effect of confinements (e.g., for unpatterned planar walls). These effects cannot be ignored, since confinements reduce the interfacial tension and thereby assist in the formation of large interfaces, leading to greater adsorption or storage of gas-like molecules on the selfassembled channels. The gases stored using such devices can be used to withdraw gases from the channels by heating nanorods placed at the channels edges (i.e., using channelrod matrix) and using mass diffusion and vacuum techniques from the voids. Though the fluid mechanics of channel flow is a very old problem (Daugherty and Franzini 1895; Massay 2001) but the effect of confinement under channels of nanosizes are very new and less understood. The viscosity of the fluids is the key parameter in the flow but the effect of confinement on the vicious properties of fluids at the very molecular scale in not well understood. 


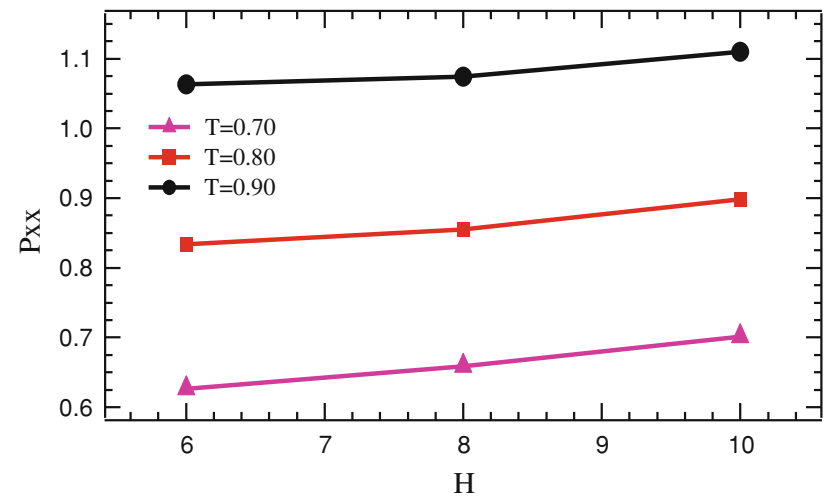

Fig. $10 X$ component of the saturated pressure Pxx versus slit width for unpatterned planar surfaces $\lambda_{\mathrm{AA}}=\lambda_{\mathrm{BB}}=\lambda_{\mathrm{AB}}=1.5$, $\varepsilon_{\mathrm{AA}}=\varepsilon_{\mathrm{BB}}=1.0, \varepsilon_{\mathrm{AB}}=-1.0, \lambda_{\text {wall-A }}=1.0, \varepsilon_{\text {wall-A }}=-1.0$. The $\mathrm{B}$ molecules have hard-sphere interactions with the walls. Curve shows the clear signature of the effect of confinement (Singh et al. 2011)

So, this model can open a new class of problems for study and the making of useful devices for the welfare of mankind.

Acknowledgments Dr S. P. Singh expresses deep and sincere thanks to Dr. Jayant K Singh and Prof. A Sharma, ChE- IITK for their generous support as supervisor and letting him to communicate the work. He also expresses sincere thanks to Department of Science and Technology, India for funding for the work under the project Grant DST/SPO/20070089.

Open Access This article is distributed under the terms of the Creative Commons Attribution License which permits any use, distribution, and reproduction in any medium, provided the original author(s) and the source are credited.

\section{References}

Allen MP, Tildesley DJ (2001) Computer simulations of liquids. Oxford University, Oxford

Bhatia SK, Myers AL (2006) Optimum conditions for adsorptive storage. Langmuir 22:1688

Cabria I, Lopez MJ, Alonso JA (2005) Enhancement of hydrogen physisorption on graphene and carbon nanotubes by $\mathrm{Li}$ doping. J Chem Phys 123:204721

Christenson HK (2001) Confinement effects on freezing and melting. J Phys Condens Matter 13:R95

Darkrim F, Levesque D (1998) Monte Carlo simulations of hydrogen adsorption in single-walled carbon nanotubes. J Chem Phys 109:4981

Daugherty RL, Franzini JB (1895) Fluid mechanics with engineering applications, 7th edn. McGraw Hill Kogakhusha Ltd., Tokyo

Dong LK (2010) Preparation and characterization of black cobalt solar selective coatings. J Korean Phys Soc 57(1):111-119. doi: $10.3938 / \mathrm{jkps} .57 .111$

Elliott JR, Hu L (1999) Vapor-liquid equilibria of square-well spheres. J Chem Phys 110:3043

Frenkel D, Smit B (1996) Understanding molecular simulation. Academic, San Deigo
Fukuzawa K, Deguchi T, Yamawaki Y, Itoh S, Muramatsu T, Zhang $\mathrm{H}$ et al (2008) Control of wettability of molecularly thin liquid films by nanostructures. Langmuir 24:2921

Gelb LD, Gubbins KE, Radhakrishanan R, Bartkowiak MS (1999) Phase separation in confined systems. Rep Prog Phys 62:1573

Green DG, Jackson G, de Miguel E, Rull LF (1994) Vapor-liquid and liquid-liquid phase equilibria of mixtures containing square-well molecules by Gibbs ensemble Monte Carlo simulation. J Chem Phys 101:3190

Grosfils P, Lutsko JF (2009) Dependence of the liquid-vapor surface tension on the range of interaction: A test of the law of corresponding states. J Chem Phys 130:054703-1

Herrera ED, Santiago GR, Moreno-Razo JA (2005) Phase and interfacial behavior of partially miscible symmetric LennardJones binary mixtures. J Chem Phys 123:184507-1

Kargupta K, Sharma A (2002) Dewetting of thin films on periodic physically and chemically patterned surfaces. Langmuir 18:1893

Katumba G et al (2008a) FTIR and Raman spectroscopy of carbon nanoparticles in $\mathrm{SiO}_{2}, \mathrm{ZnO}$ and $\mathrm{NiO}$ matrices. Nanoscale Res Lett 3(11):421-426. doi:10.1007/s11671-008-9172-y

Katumba G, Olumekor L, Forbes A et al (2008b) Optical, thermal and structural characteristics of carbon nanoparticles embedded in $\mathrm{ZnO}$ and $\mathrm{NiO}$ as selective solar absorbers. Sol Energy Mater Sol Cells 92(10):1285-1292. doi:10.1016/j.solmat.2008.04.023

Lipowsky R (2001) Wetting morphologies at structured surfaces. Curr Opin Colloid Interface Sci 6:40

Massay B (2001) Mechanics of fluids, 7th edn. Spon Press, Taylor and Francis Group, London

Orea P, Duda Y, Alejandrea J (2003) Surface tension of a square well fluid. J Chem Phys 118:5635

Orea P, Duda Y, Weiss VC, Schroer W, Alejandre J et al (2004) Liquid-vapor interface of square-well fluids of variable interaction range. J Chem Phys 120:11754

Overduin SD, Patey GN (2002) Forces between chemically patterned plates immersed in binary liquid mixtures. J Chem Physics 117:3391

Porcheron F, Monson PA, Schoen M (2006) Wetting of nanopatterned rings on solid surfaces. Phys Rev E 73:041603-1

Puri S (2007) Interplay of wetting and phase separation at surfaces. Phys A 384:100

Rocken P, Tarazona P (1996) Capillary condensation in structured pores. J Chem Phys 105(5):2034

Rother G, Woywod D, Schoen M, Findenegg GH et al (2004) Confinement effect on the adsorption from a binary liquid system near liquid/liquid phase separation. J Chem Phys 120: 11864

Roussel T, Bichara C, Gubbins KE, Pellenq RJ-M (2009) Hydrogen storage enhanced in Li-doped carbon replica of zeolites: A possible route to achieve fuel cell demand. J Chem Phys 130:174717-1

Schoen M (2008) Fluid bridges confined between chemically nanopatterned solid substrates. Phys Chem Chem Phys 10:223

Singh SP (2008) Theory of Spinodal Dewetting of thin films: MC simulations with a special reference of surface directed phase separation, Chapter-I. Ph.D thesis, Department of Physics and Electronics, Dr. R M L Avadh University, Faizabad, UP, India

Singh JK, Kofke DA (2005) Molecular simulation study of the effect of pressure on the vapor-liquid interface of the square-well fluid. Langmuir 21:4218

Singh JK, Kofke DA, Errington JR (2003) Surface tension and vaporliquid phase coexistence of the square-well fluid. J Chem Phys 119:3405

Singh SP, Singh JK, Sharma A (2011) Investigating bridge like structures in a square well binary mixture using NVT Monte Carlo simulation. Paper presented in International conference on advanced nanomaterials and nanotechnology 9-11 Dec. 2009, 
Indian Institute of Technology Guwahati, India. Special Issue of Int J Nanosci 10(I):329-333

Steiner U, Klein J (1996) Growth of wetting layers from liquid mixtures. Phys Rev Lett 77:2526

Trokhymchuk A, Alejandre J (1999) Computer simulations of liquid/ vapor interface in Lennard-Jones fluids: some questions and answers. J Chem Phys 111:8510
Yan LT, Li J, Li Y, Xie X-M et al (2008) Kinetic pathway of patterndirected phase separation in binary polymer mixture films. Macromolecules 41:3605

Yuan Y-Q et al (2004) Effect of the interaction strength and range on the wetting behavior in confined binary fluid. Phys Lett A 321:388 